\title{
LES EFFETS DE LA MONDIALISATION SUR LA GOUVERNANCE
}

\author{
OS EFEITOS DA GLOBALIZAÇÃO NA GOVERNANÇA
}

THE EFFECTS OF GLOBALIZATION ON GOVERNANCE

\section{Grace Ladeira Garbaccio}

\begin{abstract}
Professora do Programa Stricto Sensu do Mestrado Acadêmico em Direito e do Mestrado Profissional em Administração Pública do IDP. Doutora e mestre em Direito pela Universidade de Limoges/ França - reconhecido pela Universidade Federal de Santa Catarina (UFSC). Professora do curso de pós-graduação lato sensu da ESPM, EDB e FMU. Gestora de sustentabilidade e comunicação em empresas multinacionais e nacionais.

E-mail: glgarbaccio@ hotmail.com
\end{abstract}

\section{Marie Tanchon}

Professionnelle de recherche à la Chaire de recherche et d'innovation Goldcorp en droit des ressources naturelles et de l'énergie. Elle est titulaire d'un L.L.M. en droit de l'environnement, développement durable et sécurité alimentaire de l'Université Laval, Québec, Canada et d'une maîtrise en droit de l'environnement de l'Université Paris 1

Panthéon- Sorbonne, Paris, France. Ses recherches portent principalement sur le droit et l'acceptabilité sociale des projets miniers mais aussi, de manière générale, en droit de l'environnement. Elle est membre chercheur de l'Institut Hydro-Québec en environnement, développement et société (Institut EDS). E-mail: marie.tanchon.1@ulaval.ca

Recebido em: 04/04/2020

Aprovado em: 10/11/2020

RÉSUMÉ: Dans le contexte global, la mondialisation entraîne plusieurs impacts sur la notion de gouvernance. Cette notion sera traitée tout au long de cet article de manière conceptuelle et politique par l'étude des enjeux fondamentaux qu'elle abrite et par la proposition de pistes d'action dans le domaine de la gouvernance mondiale. Dans un premier temps, l'analyse portera sur le contexte et le terme même de mondialisation. Celle-ci se fera sous les traits de l'échelle mondiale de la réussite et de modernité comme références. Les possibilités de préserver la singularité culturelle face à la mutation de la société internationale seront, par la suite, évoquées par le prisme de la défense d'une protection interne et par celui de la mobilité comme phénomène d'analyse de la mondialisation. Dans un second temps, les notions de gouvernance et de mondialisation seront confrontées afin de se questionner sur les possibilités d'une gouvernance mondiale adaptée aux enjeux du XXIème siècle. À travers une synthèse scientifique, cet article utilise la méthode de la recherche déductive et exploratoire, basée sur l'analyse des références d'articles et des études bibliographiques.

Mots-clés: Gouvernance mondiale. Mondialisation. Libre-échange. Singularité et uniformisation culturelle. Principe de mobilité.

RESUMO: No contexto mundial, a globalização conduz a vários impactos no conceito de 
governança. Essa noção será tratada ao longo deste artigo de maneira conceitual e política, estudando os desafios fundamentais inerentes e propondo indicações no campo da governança global. Primeiro, a análise se concentrará no contexto e na própria expressão de globalização. A mesma será abordada em escala global de sucesso e modernidade como referências. As possibilidades de preservar a singularidade cultural, diante da transformação da sociedade internacional, serão evocadas pela ótica da defesa da proteção interna e pela mobilidade como um fenômeno de análise da globalização. Numa segunda etapa, serão confrontadas as noções de governança e globalização, a fim de se questionar as possibilidades de uma governança global adaptada aos desafios do século XXI. Através de uma síntese científica, este artigo utiliza o método de pesquisa dedutiva e exploratória, com base na análise de referências de artigos e estudos bibliográficos.

Palavras-chave: Governança mundial. Globalização. Livre comércio. Singularidade e uniformização cultural. Princípio da mobilidade.

ABSTRACT: In the global context, globalization leads to several impacts on the concept of governance. This notion will be treated throughout this article in a conceptual and political way, studying the inherent fundamental challenges and proposing indications in the field of global governance. First, the analysis will focus on the context and the very expression of globalization. It will be addressed on a global scale of success and modernity as references. The possibilities of preserving cultural uniqueness, in the face of the transformation of international society, will be evoked from the perspective of defending internal protection and mobility as a phenomenon of analysis of globalization. In a second stage, the notions of governance and globalization will be confronted, in order to question the possibilities of global governance adapted to the challenges of the 21 st century. Through a scientific synthesis, this article uses the method of deductive and exploratory research, based on the analysis of references of articles and bibliographic studies.

Keywords: Global governance. Globalization. Free trade. Singularity and cultural uniformity. Principle of mobility.

SUMÁRIO: Introduction. 1 Le contexte de la mondialisation. 2 L'acceptabilite de la mondialisation : pourrait-elle subir un ralentissement? 2.1 L'échelle mondiale de réussite. 2.2 La modernité comme référence d'analyse de la mondialisation. 3 Est-il possible de préserver la singularité culturelle face à la mutation de la société internationale ? 3.1 La défense d'une protection interne. 3.2 La mobilité comme phénomène d'analyse de la mondialisation. 4 Une gouvernance mondiale, est-elle possible au XXIème siècle ? Conclusion. Bibliographie.

\section{INTRODUÇÃO}

Le concept de gouvernance est à la mode. La mondialisation est un terme récent mais le phénomène est ancien. Ce sont les grandes découvertes au XVIème siècle qui ont ouvert la voie. Aujourd'hui, les échanges sont de plus en plus denses et variés : flux commerciaux, humains, culturels, etc. Sa diffusion n'épargne aucun échelon de l'action collective, qu'elle soit privée ou publique. « On parle de gouvernance d'entreprise, de gouvernance locale et, bien entendu, de gouvernance mondiale ». Conceptuellement, cela veut dire analyser les enjeux fondamentaux qu'il abrite. Politiquement, cela signifie avancer des pistes d'action dans le domaine qui nous concerne ici : la gouvernance mondiale. Le concept de gouvernance trouve probablement son origine dans la théorie de l'entreprise (WILLIAMSON, 1975 ; BEVIR, 2011). La gouvernance y apparaît comme une théorie des coûts de transaction qu'une entreprise doit faire entre internalisation et externalisation de la sous-traitance. L'idée de gouvernance renvoie donc en premier lieu à l'idée 
essentielle d'arbitrage entre des choix stratégiques qui garantissent soit le contrôle au détriment du coût (internalisation), soit l'inverse (externalisation) (LAÏDI, 2020).

Ce sont essentiellement le libre-échange et les progrès dans les domaines des transports et télécommunications qui ont permis l'accélération de la mondialisation constatée depuis les années 80 (BEVIR, 2010). De plus, peu de régions dans le monde sont à l'écart de la globalisation. En outre, ce processus engendre des réactions et des résistances entre populations qui tentent d'imposer leurs propres règles.

Selon SÈVES (2003) et BEVIR (2010), la mondialisation commence avec l'expansion coloniale de l'occident. Après avec un mouvement contraire aboutissant les peuples à l'indépendance, entraînant la division du monde en blocs. C'est seulement après la chute du mur de Berlin et l'éclatement de l'URSS, que la mondialisation, comme système de relations sociales comprenant tous les peuples, peut se manifester dans son intégrité, indépendamment d'un antagonisme fondamental reflétant des visions du monde inversées.

La mondialisation, juridiquement, serait la réalisation tardive de la société internationale, avec la relation harmonieuse d'Etats souverains.

Sur le plan économique, la division nord-sud, au cœur des revendications du nouvel ordre économique international, promu par l'ONU des années 70, a laissé la place à des phénomènes complexes de rattrapage et de division non seulement sud/sud, mais dans une moindre mesure nord/nord, qui iront sans doute en s'accentuant, notamment parce que la pression économique de la Chine s'exerce non seulement sur les industries du nord mais également sur ses concurrents du sud - Brésil, Mexique, Thaïlande, Indonésie, etc. - une tendance que le démantèlement de l'Accord Multi-Fibres en 2005 ne manquera pas d'accentuer dans le secteur capital du textile (SÈVE, 2003, p. 4).

C'est aussi l'existence même de tels blocs idéologiques et souvent géographiques, résurgence de l'historicisme hégélien, qui fait problème. Comme l'indique Jean-Pierre WARNIER (1999, p. 99) le «vrai problème auquel les sociétés contemporaines sont confrontées est un problème d'éclatement et de dispersion des références culturelles plus que d'homogénéisation ».

Le modèle classique de la société internationale interétatique néglige en effet les conditions matérielles et sociaux nécessaires à la constitution d'un Etat, ce que l'on pourrait appeler le coût de l'Etat de droit. Selon SÈVE (2003) et ABI KHALLIL (2015), la solution la plus envisagée consiste à promouvoir des mécanismes d'intégration régionale, afin de mutualiser certains coûts de souveraineté, mais elle reste difficile à mettre en œuvre, sans intervention extérieure, lorsque chacun se débat ou s'enfonce dans les mêmes difficultés, ou lorsque certains protagonistes espèrent en tirer profit.

Il est d'abord loin de remplir toujours avec efficacité les tests politiques et économiques que l'on reproche au droit international de ne pas maîtriser, sous l'effet d'une criminalité internationale qui le défie sur son territoire ou plus généralement d'une mobilité de certains acteurs qui leur permet d'échapper à son autorité. Le droit interne, quand il ne transpose pas le droit international, à moins encore qu'il n'évolue, non par assimilation au droit international comme tel, mais par réception du droit interne des autres nations envisagées certes à l'aube de leur caractère civilisé mais également à celle de leur compétitivité.

Inversement, le droit international s'est rapproché du droit interne dans la mesure où, même s'il garde des dimensions spécifiques (le maintien de la paix, le droit de la guerre, le contrôle des armements...), il s'est étendu à des problématiques économiques, écologiques, sociales, humanitaires (KAMTO, 2015). Le Protocole de Kyoto est un exemple de cette réalité.

Mais le droit international partage les mêmes interrogations que le droit interne sur sa démarche institutionnelle, sur la frontière entre public et privé, la distinction du public et de l'étatique, la problématique de la gouvernance et du contrôle, etc.

«On dit souvent que la maturité fait apparaître la politique comme dilemme alors que la jeunesse l'a découverte comme scandale. Retournons la proposition au nom du réalisme actif : pour 
aider à résoudre plus vite les dilemmes, conservons donc l'esprit d'indignation devant les scandales »(GEORGE, 2002, p.66).

Le problème de recherche auquel cet article tente de répondre porte sur l'identification et l'analyse des effets de la mondialisation sur la gouvernance mondiale, notamment sous son triple aspect économique, social et politique. Partant de cette problématique, l'hypothèse de recherche est la suivante : la diversité et la multiplication des acteurs, à la fois institutionnels et privés, renforcent la légitimité de la régulation de la gouvernance mondiale. Toutefois, l'acceptabilité de la mondialisation actuelle reste largement conditionnée par la participation effective de chacun de ces acteurs au processus décisionnel et juridictionnel. Afin de démontrer la validité de cette hypothèse, quatre aspects des effets de la mondialisation sur la gouvernance mondiale seront analysés. Premièrement, le contexte de la mondialisation sera analysé. Deuxièmement, nous nous intéresserons à la notion d'acceptabilité de la mondialisation et nous nous questionnerons sur la prévisibilité d'un ralentissement. Troisièmement, nous confronterons la notion de singularité culturelle face à la mutation de la mutation de la société internationale. Enfin, nous analyserons les possibilités d'une gouvernance mondiale au XXIe siècle.

À travers une synthèse scientifique, la méthode de la recherche employée est la déductive et exploratoire, basée sur l'analyse des références d'articles et des études bibliographiques.

\section{LE CONTEXTE DE LA MONDIALISATION}

La crise environnementale a rendu encore plus manifeste la dimension internationale des problèmes environnementaux, dimension qui lui préexistait, mais que l'on pouvait appréhender comme l'échappatoire lointain de problèmes qui se traitaient essentiellement au niveau national. Cela n'est plus possible. Les phénomènes naturels ignorent les frontières.

Les questions relatives à l'émission de GES supposent des procédures de négociations internationales qui permettent à la fois d'édicter des mesures et de prendre en considération les intérêts légitimes des différents pays. Le traitement international de la crise environnementale est rendu possible par l'internationalisation croissante de la vie politique, à laquelle nous assistons. Parallèlement, la mondialisation de l'économie renforce considérablement la réalité de l'international, comme une instance ayant une existence propre.

La polarisation politique de la crise environnementale a, au niveau international, des effets comparables à ceux du sociocentrisme à l'intérieur d'une communauté politique : la dimension politique fait disparaître la dimension environnementale. Remédier à la crise apparaît ainsi comme un luxe de riche que le Nord voudrait imposer au Sud, en bloquant un développement dont le sud a besoin et que le nord a atteint depuis longtemps : le rapport à la nature disparaît dans la contradiction entre pays pauvres et pays riches.

La notion de développement durable peut se révéler comme une tentative d'échapper à cette réduction, en tentant d'articuler la nature et la politique. L'acceptabilité de la mondialisation est-elle liée à son ralentissement ?

\section{L'ACCEPTABILITE DE LA MONDIALISATION : POURRAIT-ELLE SUBIR UN RALENTISSEMENT ?}

Bien que la globalisation soit incontournable, elle demeure inachevée étant donné qu'elle ne concerne pas toutes les régions du monde. Certes les acteurs permettant l'intégration à l'espace mondialisé sont divers, on compte parmi eux les firmes multinationales, les Etats et les organisations régionales, cependant, certains pays demeurent tenus à l'écart de la mondialisation (ABI KHALLIL, 2015). En effet, certains espaces émergent, ce sont ceux qui sont à l'origine des différents flux, émetteurs et récepteurs : il s'agit des pays de la triade et plus précisément les façades maritimes et les grandes métropoles de celle-ci. D'autres au contraire, cherchent à tout prix à 
s'intégrer à ce processus espérant par la rejoindre la voie du développement : ce sont majoritairement les pays nouvellement industrialisés, particulièrement ceux d'Asie du sud-est, ainsi que la Chine. Les pays les plus pauvres, enfin sont complètement délaissés, ce qui expliquent - mais ne justifient pas pour autant - certaines réactions des groupes alter mondialistes et terroristes antiaméricains.

Les résistances à la mondialisation économique fondent leur argumentation sur les deux éléments suivants:

- d'abord sur les effets négatifs des délocalisations :

1. qu'elles touchent les pays du nord : les délocalisations sont à l'origine de fermetures d'usines, de suppressions d'emplois dans les pays du Nord (citons à cet effet l'exemple du secteur français du textile),

2. ou ceux du sud : les conditions de travail sont précaires, dangereuses pour les employés.

- puis sur les déséquilibres du commerce international. Ces inégalités ayant entraîné de vives réactions de la part des altermondialistes. Au Nord comme au Sud, des groupes de pression se multiplient comme Association pour la taxation des transactions pour l'aide aux citoyens (ATTAC), Confédération paysanne qui organisent depuis quelques années des Forum Social Mondial (FSM) comme ceux de Porto Alegre / Brésil ou de Bombay.

Toutefois, cela n'a pas empêché que la mondialisation économique prenne pleinement place dans notre réalité.

\subsection{L’échelle mondiale de réussite}

Le processus d'unification prend le nom de mondialisation. Ce mot «mondialisation » est souvent utilisé de façon péjorative pour désigner le processus d'intégration économique des divers pays de la planète sous le libéralisme (MATTEI, 2003). Nous proposons d'établir dans cette communication, que le procès de mondialisation, sous son triple aspect économique, social et politique, loin d'être destructeur de l'humain, répond à une rationalité implicite qui sécrète, au sens premier du terme, une éthique de liberté universelle.

L'avènement du monde à partir de l'œuvre est fondamentalement la perte de l'humain si l'on entend par « humain » le repli sur l'utilisation du produit exprimée par sa valeur d'usage, et, au-delà encore, par sa valeur d'échange.

L'économiste américain Théodore LEVITT en 1983 a introduit le terme globalisation ou « globalization » dans « The Globalization of Markets », Harvard Business Review en mai juin 1983, pour désigner la convergence des marchés offerts aux produits des grandes sociétés multinationales. Le terme a été popularisé en 1990 avec la publication du livre de Kenichi OHMAE - «The Bordless World : power and strategy in the interlinked economy ».

Selon MATTEI (2003), la mondialisation risque de nous faire perdre le monde, non parce que le monde s'est retiré des échanges économiques, mais parce que l'homme est apparu sur la scène sous les aspects du sujet, du producteur et du consommateur.

La place du monde est occupée par un espace d'échanges où vont et viennent les produits, les services et les hommes, c'est-à-dire un réseau syntaxique complètement internationalisé.

L'automouvement de la subjectivité, plus profondément ancrée dans son désir que dans sa volonté, devient l'automouvement de l'économie, car l'économie est l'espace matriciel du mouvement : mouvements de capitaux, des transferts de services, des délocalisations de productions, des échanges de produits, des réseaux d'informations. S'il est vrai que, " ontologiquement, la modernité est un pur être vers le mouvement » (SLOTERDIJK , 2000, p.36), ce qu'avait déjà politiquement observé Tocqueville de la démocratie, la mondialisation est un pur être vers la mobilisation. «Autrement dit, la mondialisation est le processus fondamental de la modernité comme l'avait pressenti JUNGER, dès 1932, avec sa théorie de la mobilisation dans la figure du travailleur, le sujet planétaire qui a perdu son monde et, avec son monde, sans doute son 
âme » (MATTEI, 2003, p.116).

Le processus de mondialisation se confond aujourd'hui avec le processus de la vie économique. Pour les critiques de l'économie libérale, le professionnel du marché marque la fin du monde dès lors que nous réduisons effectivement ce monde que nous habitons au cycle vital de la production et de la consommation.

Le processus de mondialisation, compris essentiellement comme " circulation », ne se réduit pas à l'opposition simpliste entre culture dominante et culture dominée. Il est bien plutôt « une mise en mouvement de cultures qui s'interpénètrent » de telle sorte que « la mondialisation engendre davantage d'hétérogénéité, de différences, que d'uniformité et de ressemblances » dans cet espace d'échanges qu'il qualifie d'hyper démocratie » (MATTEI, 2003, p.118).

Concernant son universalisation, si la mondialisation est bien l'horizon nécessaire de notre temps, il n'est pas possible de trouver un point de vue, et un point d'appui, hors de ce cadre global pour le transformer ou le supprimer. Archimède, et après lui Descartes, demandait un point d'appui hors du monde pour soulever le monde, ce qui est par définition impossible. Personne ne saurait trouver un point d'appui hors de la mondialisation pour changer la mondialisation, c'est-à-dire pour changer le processus de constitution du monde. Nous ne changerons jamais la caverne, ni ne la supprimerons en nous en évadant. C'est la caverne, entendons le monde lui-même, qui changera à son rythme, et, en changeant ainsi, changera la totalité de l'humanité.

Concernant sa rationalisation, « la mondialisation des sociétés humaines ne signifie rien d'autre que l' " universalisation » de leurs principes, de leurs méthodes et de leurs techniques. Le mondial, ou théorique, l'universel, c'est le principe d'unité ou, mieux, d'unification, qui commande ce processus historique sans que les multiplicités soient affectées en tant que multiplicités. La mondialisation ne met pas fin aux singularités culturelles et sociales; elle leur donne au contraire de nouvelles scènes, de nouveaux masques et de nouveaux statuts. On ne peut jouer la raison contre la raison, et la mondialisation contre la mondialisation, sans succomber à une contradiction interne qui laisse intactes, à la fois la mondialisation, comme processus effectif des échanges et la rationalisation, comme opérateur unique de ce processus effectif » (MATTEI, 2003, p.118-119).

Concernant sa libéralisation, le mode d'expression de l'universel, qui se déploie concrètement dans la mondialisation, est l'échange. Le terrain naturel des processus incessants de mondialisation est le marché. Or, l'économie est une modalité spécifique de l'échange appliquée aux produits du travail humain, non plus échange de raison, mais échange de biens et de services sur fond d'engagement réciproque. A ce titre, tout échange économique est, du fait de sa source rationnelle, universalisable, ou, si l'on préfère, indéfiniment ouvert. Et le marché n'est rien d'autre que le système global des informations ou des services que les hommes produisent et échangent à tout moment. Dans son principe aussi bien que dans son processus, le marché apparaît comme un système rationnel, aussi rationnel que le langage, quand bien même sa rationalité, à l'image de celle du monde, demeurerait cachée. S'il y a un ordre économique possible, comme le reconnaissent ceux qui dénoncent sa forme capitalistique, un ordre économique qui est lié à l'ordre politique et à l'ordre juridique, cet ordre ne peut être fondé que sur la liberté des agents économiques. Il ne saurait en conséquence être limité à l'intérieur des frontières nationales. Un ordre économique mondial, fondé sur la liberté des échanges, doit accompagner un ordre politique mondial, manifesté par l'horizon démocratique et fondé sur la liberté des citoyens.

Selon MATTEI (2003), si la mondialisation est la transformation, non pas du monde, mais de son aspect économique en une zone de libre-échange, elle ne se confondra pas avec la marchandisation. Pensée dans son principe, elle est le processus d'identification de l'homme qui répond, dans le champ économique, à son universalisation théorique en tant que modèle rationnel. La mondialisation du monde, c'est la réalisation de la réalité, ou la réalisation de tout ce qui, dans les échanges entre les hommes, qu'il s'agisse de paroles, de biens et de services, en un mot, d'informations, ne peut s'effectuer que librement.

Ce raisonnement nous amène à penser la modernité comme moyen de compréhension de 
la mondialisation. C'est ce que nous proposons analyser dans la partie suivante.

\subsection{La modernité comme référence d'analyse de la mondialisation}

« Le concept adopté par HABERMAS sur le moderne/modernisation concerne l'accumulation : selon ce philosophe modernisation concerne l'accumulation de capital et la mobilisation de ressources ; les développement de forces productives et l'augmentation de la productivité du travail ; l'imposition d'une violente politique centrale institutionnalisée et la formation d'identités nationales ; l'expansion du droit de participation politique, de la façon de vie urbanisée ; la sécularisation de la valeur et de la norme » (MATTEI, 2003, p.122).

D'une part, la mondialisation ne se confond pas avec le processus de modernisation car elle en n'est qu'un moment particulier. D'autre part, elle ne rime pas obligatoirement avec uniformisation. Selon YATABE (2003), au contraire, c'est le mouvement qui fait basculer la société japonaise d'un univers d'un seul tenant à un monde multiforme, le passage d'un espace géographique et social perçu comme « une île » à un espace vécu et ressenti comme « un archipel ", qui ouvre à la mondialisation l'espace au sein duquel elle vient se blottir. Ces mutations accompagnent l'effacement de la dynamique intégrative, qui était celle du Japon durant les années de croissance économique, au profit d'une forte propension à la différenciation sociale, dont un des effets les plus manifestes, à l'instar d'autres sociétés modernes, est l'emballement du processus d'individualisation inhérent à la modernité industrielle.

Sa théorie peut donc admettre que ce qui avait pu constituer un progrès au cours de l'histoire ait pu régresser par la suite. Ainsi F.A.von HAYEK parle de la " démocratie dégénérée du temps de Platon » (DE SALLE, 2003, p. 153).

« Dans l'attente d'une restauration du processus, plusieurs contraintes s'imposent à nous. Entre autres, celle consistant à progresser à un rythme soutenu si nous voulons préserver la paix dans le monde et la civilisation. Les aspirations de la grande masse de la population ne peuvent effectivement être satisfaites que grâce à un progrès matériel rapide. Pour Hayek, ceci implique une longue phase d'avancées matérielles » (DE SALLE, 2003, p. 153).

Face à ces avancées matérielles, nous nous interrogeons sur la possibilité de préserver une singularité culturelle avec tout un processus de mutation de notre société actuelle.

\section{EST-IL POSSIBLE DE PRÉSERVER LA SINGULARITÉ CULTURELLE FACE À LA MUTATION DE LA SOCIÉTÉ INTERNATIONALE?}

Selon CHENET (2003), c'est dire combien la mondialisation apparaît prima face comme un processus multiforme et complexe, de par la superposition de mouvements qu'elle enveloppe, l'évaluation de son incidence qui s'avère délicate, tant ses conséquences se révèlent souvent ambiguës, voire contradictoires, et d'autant plus malaisées à apprécier que le phénomène de la mondialisation s'accompagne d'une série d'hystérésis.

$\mathrm{Au}$ plan purement économique, la mondialisation assurerait la réalisation à l'échelle planétaire d'une division internationale du travail efficace, créatrice de richesses, conforme à la théorie des avantages comparatifs. S'il est vrai que le marché fabrique de l'efficacité et de l'inégalité, et ce d'autant plus que la techno science est de nos jours largement mise au service d'une économie de concurrence, la question est alors moins de déplorer le fait que la mondialisation génère certes son lot d'injustices que de déterminer avec précision si elle contribue ou non à élever le niveau de vie moyen d'un pays.

La mondialisation a des conséquences équivoques sur le plan social. Elle entraîne, en effet, des changements de valeurs, favorise les transformations dans les modes de vie et l'épanouissement par là même de la démocratie libérale - régime impliquant une conception contractuelle du droit amène à l'autonomie des producteurs et des consommateurs, à l'individualisme, à l'égalitarisme, 
au rationalisme et à la sécularisation des visions de l'histoire. Ainsi, la mondialisation, semble affaiblir les croyances traditionnelles et ébranler les valeurs spirituelles et a pour effet la sécularisation des rapports sociaux. De par la perte des racines traditionnelles, les identités sociales sont de plus en plus changeantes.

Selon LE CACHEUX (2002), c'est ainsi que la mondialisation recouvre un ensemble d'évolutions dont certaines ne sont pas récentes, mais qui toutes se sont toutes accélérées au cours des deux décennies passées, évolutions qui toutes s'incarnent fort concrètement, même quand elles ne sont pas aisément descriptibles dans leurs mécanismes ou leurs causes.

Que la mondialisation soit un état de fait, qu'elle soit une dynamique historique irréversible et irrépressible, elle est de toute manière l'évidence.

L'uniformisation culturelle - constatée depuis 1945 - à partir du modèle occidental, particulièrement celui des Etats-Unis, s'explique aisément par l'absence d'identité propre à ce pays. Issus de l'immigration mondiale, les Etats-Unis sont le produit d'un métissage entre culture africaine, européenne, hispanique, etc., le facteur commun entre toutes ses cultures étant la langue dominante : l'anglais. Actuellement, les pays de l'est se réclament de cette culture.

Selon De SALLE (2003), F.A.von Hayek défendait l'idée selon laquelle la tradition produit certains mécanismes - fondement de la démocratie et du libéralisme - ceux-ci doivent tout à l'histoire qui aurait pu les rendre différents de ce qu'ils sont.

Il précise bien que les mécanismes de l'évolution culturelle sont totalement différents de ceux de l'évolution naturelle. Ni Darwin ni F.A.von Hayek n'ont inventé le concept d'évolution. En effet, des philosophes moralistes du XVIIIème siècle, avaient déjà formulé, dans leur discours sur le droit, la morale et la monnaie..., des notions similaires à celles d'évolution et de formation spontanée. De plus, il serait préjudiciable de croire que la théorie de l'évolution se résume à des « lois de l'évolution ». Cette théorie à proprement parler, n'est que la description d'un processus dont le résultat dépend étroitement d'une multitude de facteurs, si bien qu'il est difficile d'en tirer des prévisions quant au futur.

La sélection, qu'elle soit culturelle ou naturelle, est un processus concurrentiel. Les structures issues des pratiques traditionnelles humaines ne sont quant à elles ni naturelles c'est-àdire engendrées par un processus biologiquement déterminé, ni artificielles, soit produites par un dessein intelligent.

F.A.von Hayek montre par-là que les traditions erronées ne peuvent donc en aucun cas, engendrer une morale valide ou non. C'est vraisemblablement, d'après lui, le privilège exclusif de la société libérale. La tradition occidentale a été précédée par la mentalité tribale, celle de la « société fermée » ou «société du face-à-face ». Il affirme que nos obligations morales sont engendrées par un ordre reposant lui-même sur certaines règles. Ainsi, une société ne peut subsister qu'à la condition qu'une quelconque pression amène les membres à se conformer aux règles en question.

Nous pouvons citer certaines résistances vers la mondialisation culturelle. Il faut remarquer qu'elles ne concernent pas exclusivement la mondialisation comme motif d'encouragement : des résistances pacifiques -au sein de l'aire occidentale avec par exemple l'exception culturelle en France, les actions de José Bové contre les Mac Donald ; des États qui interdisent ou limitent l'accès à internet prouve bien qu'ils résistent à la culture monde, comme Cuba, Corée du nord, Arabie Saoudite ; et des réactions plus violentes : attentats antiaméricains notamment ceux du 11 septembre 2001.

Ces résistances nous amènent au développement de moyens pour combattre ou pour minimiser ses effets. La protection interne du marché, de l'emploi, de la culture, etc. est un bon exemple de cette démarche. Passons, à l'analyse de cette protection interne. 


\subsection{La déefense d'une protection interne}

La mondialisation est souvent présentée comme un phénomène économique, se réduisant, pour l'essentiel, à l'augmentation spectaculaire des flux de capitaux depuis les années 1970. Or la mondialisation, processus toujours en cours de dynamiques pluriséculaires, est un mouvement global, impliquant la totalité de la terre, ainsi que toutes les dimensions de l'homme. La mondialisation peut être définie comme une intensification considérable des flux de toutes sortes (marchandises, services, idées, modes, cultures...) se traduisant par une contraction massive de l'espace et du temps - rapidité des communications, effondrement des coûts de transport... (BOQUET, 2018 ; CARROUÉ, 2018).

Selon DEFARGES (2003), la mondialisation est un phénomène politique. Au moment même où, à la fin du XVIIIème siècle, la révolution industrielle sème les germes d'une exploitation méthodique de toutes les ressources terrestres et rend matériellement possible un maillage beaucoup plus dense de la planète, la révolution démocratique prend racine. La difficulté centrale, pour les institutions mondiales, c'est qu'elles sont vouées à vivre dans deux univers à la fois, univers tout autant opposés qu'enchevêtrés. Le premier univers, hérité de l'histoire, est celui dit de la jungle, milieu traditionnel des relations internationales, régi par des rapports de brutalité et gouverné par les plus forts. Le second univers est un vaste chantier, visant à construire une société mondiale, dotée de normes explicites et d'un policier institutionnel.

La facilité par laquelle nous sommes mobiles actuellement est un facteur important d'analyse de la mondialisation.

\subsection{La mobilitée comme phénomène d'analyse de la mondialisation}

Selon FRISON-ROCHE (2003), deux types de mondialisations peuvent être identifiées :

- la première se traduit par l'intensification de la mobilité à travers les frontières ; à celleci doit répondre une rapide et nécessaire évolution d'un droit qui demeure gouverné par la distinction du mobile et de l'immobile.

- la seconde est le résultat de la création d'espaces virtuels, sans territoire ni accroche matérielle.

L'auteure aussi explique que l'internationalisation peut être désignée comme l'arrachement des personnes et des organisations au sol par le phénomène de la mobilité. Ce principe de mobilité s'est cristallisé en droit par le droit international, propre aux phénomènes qui passent les frontières mais qui ne détruisent pas celles-ci. Si la mondialisation se résume dans l'intensification de cette mobilité, par l'accélération et l'amplification des déplacements des personnes et des choses, il faut alors certes infléchir le droit international mais celui-ci, dans sa nature, demeure pertinent (KAMTO, 2015).

L'Europe doit garder son regard tourné vers l'extérieur et non vers l'intérieur. Elle doit centrer ses efforts sur la compétition extérieure et adapter son modèle social pour pouvoir combiner flexibilité et équité. En effet, nous devons être conscients que la Chine et l'Inde, non seulement sont à la pointe du progrès en ce qui concerne les biens à faible valeur ajoutée et les services peu qualifiés, mais fournissent quatre millions de diplômés par an. L'Europe doit par des réformes économiques intensifier ses efforts dans les biens et services hautement qualifiés.

La réponse est que l'Europe est en train de tenter l'impossible, et qu'il est grand temps que nos responsables politiques s'en aperçoivent afin que l'on puisse débattre de la question de savoir comment remplacer ce modèle économique libéral par un système qui protège les économies, les sociétés et l'environnement dans le monde entier.

Ce qui est sidérant avec la Chine, c'est que pour la première fois nous sommes face à un pays immense, pauvre et qui peut être compétitif par ses bas salaires et sa haute technologie. Combiner les deux, pose un sérieux problème aux Etats-Unis et à l'Europe. 
Si nous croyons que la concurrence exercée par les pays émergents comme l'Inde et la Chine est simplement due à leurs bas salaires, nous nous trompons gravement. Ces pays deviennent compétitifs dans des secteurs hautement technologiques parce qu'ils investissent de plus en plus dans la recherche et l'innovation.

On fait des pressions constantes sur l'Europe pour qu'elle imite « la success story » américaine avec ses marchés ouverts, sa flexibilité (moins de travailleurs), sa faible bureaucratie (régulation) pour être compétitive contre l'Inde et la Chine.

Les concurrents étrangers se plaignent de ce que la compétitivité chinoise est fondée sur des pratiques sociales et environnementales injustes.

Si rien n'est fait, la Chine va émettre de plus en plus de $\mathrm{CO} 2$ ces vingt prochaines années et va devenir, devant les Etats-Unis, le premier émetteur mondial de GES, ce qui rendra par contraste ridicules les efforts faits par les autres pays pour diminuer leurs émissions.

La Chine n'est pas en soi le problème, elle n'est que le symptôme de l'échec du système commercial mondial. Même si la Chine semble gagnante au vu des chiffres de sa croissance et de ses exportations, en réalité le système bénéficie aux multinationales et coûte au plus grand nombre.

Pour s'en sortir, il faut "relocaliser » afin de protéger les économies locales avec des mesures garde-fous comme des taxes et quotas, une politique de production et de consommation locales, plus de démocratie, et la réorientation des objectifs et des réglementations du commerce afin qu'il conduise à la reconstruction des économies locales, à des échelles variables selon la taille des pays. Une autre démarche actuelle et tout à fait cohérente face aux émissions de GES liées au transport est la possible augmentation de son coût. Cette surtaxe pourra faire évoluer la production «just in time » vu que cette pratique augmente les émissions de GES et en conséquence le coût. Nous pouvons prévoir une telle augmentation du coût du transport en fonction des changements climatiques à tel point que la réduction du coût acquise avec la délocalisation ne sera pas suffisante pour combattre le coût du transport. Ce qui entreprendra une démarche dans le sens contraire : vers une relocalisation plus proche du marché consommateur. Cette relocalisation de l'économie s'impose donc dès aujourd'hui.

Pour suivre cette réalité, devons-nous donc inventer une sorte de droit virtuel, c'est-à-dire sans rattachement au physique des choses? Il s'agirait d'un droit dont les signes ne renverraient qu'à d'autres signes.

PERELMAN (1999) a pensé le droit sans attache nécessaire au monde physique, notamment parce que les auditoires peuvent aussi se constituer et les échanges argumentés se construisent à travers l'immatérialité des médias. La façon dont le droit des espaces globaux se construit correspond singulièrement à cette conception «perelmanienne » de la juste production du droit. Ainsi, le droit des marchés financiers comme celui d'Internet est avant tout constitué d'arguments articulés entre eux, pour se consolider les uns ou les autres, ou se mettre en balance les uns avec les autres, arguments qui sont agrées, décantés et peaufinés par des auditoires concentriques allant du plus spécialisé, voire du plus secret, vers le plus profane. En cela, la rhétorique peut être un moyen d'occuper ces nouveaux espaces, d'y réinstaller « l'empire » du droit.

Cette mondialisation-là n'est plus affaire de mobilité des personnes et des choses allant de territoire en territoire puisqu'elle s'est déployée dans des espaces non territorialisés, espaces nécessairement globaux parce qu'immatériels, espaces constitués par les composants mêmes, lesquels peuvent se réduire à la notion d'information (FRISON-ROCHE, 2003).

En effet, une fois constatée l'existence de ces deux mondialisations, la première n'étant que l'intensification de l'international, avec l'existence d'une efficacité accrue d'un droit négligeant les frontières pour mieux être à l'image de l'objet qu'il prétend réguler, la seconde étant la constitution d'espaces sans rattachement, pour lequel un nouveau droit est à construire, il faut immédiatement constater que cette seconde globalisation dévore la première. En effet, à travers la finance, construite sur l'information, c'est l'économie réelle qui est affectée car ces informations 
sont elles-mêmes des biens qui permettent le financement des activités réelles (LEPELTIER, 2004). La mondialisation a pris la voie du capitalisme et celui-ci s'est déployé dans les marchés financiers. De la même façon, à travers l'information, c'est toute la connaissance qui est happée par la mondialisation, l'accès à la connaissance devenant lui-même un bien. L'économie construite sur des marchés, économie encore localisée, céderait la place à une économie construite sur des accès, technologiquement globaux.

Selon FRISON-ROCHE (2003), puisque cette mondialisation ne s'appuie pas sur la distinction du mobile et de l'immobile, distinction dans laquelle le droit avait depuis toujours ses repères, il faut trouver des nouvelles lignes de distinctions. La difficulté vient précisément de l'unicité très forte de la véritable globalisation, car toute information ressemble à une autre et les fonctionnements de marché sont analogues. Cette unicité vient aussi du fait que cette mondialisation est associée à la libéralisation et à l'adoption du principe d'économie libérale, par l'addition du libre-échange et de la libre concurrence, à travers le monde.

Le critère distinctif n'est pas le marché, en tant que le droit porterait dans certains cas des valeurs hétérogènes aux marchés et dans d'autres non, car d'une part l'économie marchande n'est pas exemple de valeurs - les valeurs peuvent se développer dans le marché - et d'autre part le droit ne peut se mécaniser au point de ne plus en porter - le droit exprime par nature des valeurs.

La distinction peut être à la fois plus politique et plus neutre, dans ce rapport entre le droit et la nature dont le droit ne doit jamais s'échapper et dans lequel il a son jeu à faire. On peut distinguer d'une part le droit qui a pour fin de favoriser les mécanismes vers lesquels la nature actuelle des choses incline mais pour lequel le droit est néanmoins requis, c'est le droit qui va permettre les échanges marchands en accroissant notamment la sécurité de ceux-ci, voire en constituant l'armature de nouveaux marchés.

Comme pour l'évolution de l'immobile au mobile (la première distinction dans une mondialisation comme forme extrême de l'internationalisation), le droit peut, dans cette seconde distinction au sein des espaces globaux, passer de l'un à l'autre, de l'invention et de la gestion des moyens à la détermination des fins. C'est ainsi que le droit qui se consolide et se conçoit chaque jour au sein de l'Organisation Mondiale du Commerce (OMC), initialement conçu comme de la première sorte, ne devra sans doute sa survie qu'en entrant dans la seconde sorte.

Une des résultantes principales du phénomène de la mondialisation libérale est certainement le rôle central des sociétés transnationales sur la scène internationale. La mutation a engendré un profond changement du régime de la protection internationale des droits de l'homme en modifiant la place et le rôle normatif des différentes sphères décisionnelles. La mondialisation économique vise avant tout l'expansion des échanges commerciaux alors que le droit international de droit de l'homme exprime une conception particulière de la mondialisation, celle d'un « universalisme éthique » où l'ONU, et indirectement l'Etat, sont considérés comme les uniques " débiteurs » des droits de l'homme et seuls capables de les protéger (ABI KHALLIL, 2015).

Nous pouvons interpréter que les codes de conduite volontaires « font partie de l'univers de l'image, de la publicité et non de celui de la légalité » et ne sont pas, par conséquent, acceptables.

La logique étatique classique de protection des droits de l'homme est ainsi bouleversée. Pour les sociétés transnationales, l'acceptation de ce nouveau rôle n'est pas purement philanthropique mais représente au contraire un commerce rentable, depuis que certains aspects de la mondialisation sont remis en cause par le militantisme citoyen. L'image de marque revêtant une importance croissante, l'idée est d'utiliser la promotion des droits économiques, sociaux et environnementaux comme une nouvelle forme de «marketing de valorisation ». Il s'agit désormais d'une réalité : la communication d'une entreprise sur l'amélioration des conditions de travail de sa main d'œuvre, des conditions de pollution, de l'utilisation des ressources naturelles, etc. lui permet à la fois d'augmenter ses résultats financiers, en renforçant la confiance du consommateur et d'éviter les opérations de boycott contre ses produits.

Face à ce bouleversement de la logique étatique classique, nous posons la question 
concernant au maintien d'une gouvernance mondiale au XXIème siècle.

\section{UNE GOUVERNANCE MONDIALE, EST-ELLE POSSIBLE AU XXIÈME SIÈCLE ?}

La chute du mur de Berlin a dissipé le brouillard qui baignait des relations internationales plus complexes que ne le laissait supposer une appréhension idéologisée et par essence réductrice.

Ce remodelage de la scène internationale se traduit par l'éclosion progressive, mais encore incertaine, d'un nouveau modèle de gouvernance se concrétisant par l'apparition de formes d'actions publiques inédites aussi bien que par l'intégration d'acteurs non étatiques (lato sensu) dans les processus d'élaboration normative.

Le champ du débat public paraît littéralement envahi par des organisations non gouvernementales (ONG) qui assument aujourd'hui un rôle polymorphe dans les relations internationales. En effet, il faut également compter avec la présence de syndicats (ouvriers, employés ou employeurs) ainsi que de groupements d'entreprises et de multinationales. Les personnes physiques sont titulaires de plus en plus de droits sans que cela ne s'accompagne d'un renforcement de leur capacité d'action.

En effet, impliquant, à des titres divers, une multiplicité d'acteurs, le processus normatif international apparaît, aujourd'hui, comme le fruit d'incessantes et inévitables négociations.

Cette volonté d'associer les acteurs non étatiques au fonctionnement de l'organisation internationale n'est pas nouvelle. L'article 71 de la Charte des Nations Unis octroyait aux ONG ce qui ne tarderait pas à devenir un véritable statut consultatif auprès du Conseil économique et social.

Selon (CHAZOURNES et MEHDI, 2005), le problème de la légitimité et des effets juridiques des standards ne réside pas seulement dans la question de savoir si les Etats parties ont adhéré ou non, ont voulu être liés ou non par lesdits standards. Il consiste aussi dans le fait de savoir comment le standard a été élaboré, c'est-à-dire qui a participé au processus d'élaboration et qui en a été exclu, et quelle expertise a été prise en compte.

Par exemple, les standards International Organisation for Standardization (ISO) sont élaborés et développés par des comités techniques comprenant des experts du secteur industriel et des milieux des échanges internationaux.

La régulation est porteuse de flexibilité et de souplesse dans l'encadrement juridique des activités et des situations. «La souplesse du droit post-moderne implique sa capacité à s'adapter à l'évolution du réel » (CHAZOURNES et MEHDI, 2005, p.35). On assiste à un accroissement exponentiel d'instruments de soft law ou de normativité dite « molle ».

Un autre point important est la forte décentralisation ou délégation du pouvoir de régulation par les gouvernements à leurs agences nationales. Ces structures sont souvent élitistes car la qualité de membre est limitée à certains pays même s'il y a parfois des consultations avec les représentants des pays non parties prenantes. Par contre, une pression politique ou économique peut être exercée pour la mise en œuvre de ces standards. Le professeur WEIL (1972, p.13) résume de fort belle manière les remous de la régulation dans le champ du droit économique : « (...) le formalisme dans l'élaboration de la règle a été jeté par-dessus bord, la précision et la stabilité de la norme ont été laissés au bord de la route, la sanction judiciaire n'accompagne plus la règle comme son ombre. Tout ce à quoi le juriste est accoutumé et attaché s'évanouit pour faire place, au nom du sacro-saint réalisme, au flou, à l'imprécis, au fuyant (...) Quoi d'étonnant à ce que, devant ces techniques déconcertantes d'un droit non conceptuel et non contraignant, " déjuridicisé » et « déjuridictionnalisé », le juriste se sent quelque peu dépaysé?».

La viabilité du phénomène «de régulation » est liée à un défi majeur : celui de sa légitimité. Un effort doit être fait pour brosser une définition de ce que devraient être la transparence et la démocratie au niveau international et quelles formes elles devraient prendre. Ainsi, les principes de non exclusion, de participation publique, d'accès à l'information ou d'équilibre pourraient garantir une régulation plus transparente et plus démocratique. 
Les initiatives les plus intéressantes des régulations environnementale ou sociale d'origine privée sont caractérisées par l'élaboration de codes de conduite ou autres labels énonçant les engagements et les principes de comportement d'une entreprise, dans des domaines tels que les relations du travail, les droits de l'homme ou la lutte contre la corruption. L'élaboration de ces nouvelles normes techniques (par exemple les normes de certification environnementale élaborées par les entreprises à destination d'autres entreprises) s'apparente au phénomène de l'autorégulation pouvant être défini comme « une technique juridique selon laquelle des règles de droit sont créées par les personnes auxquelles ces règles sont destinées à s'appliquer ». Le débat sur la valeur juridique de ces normes s'est trouvé au centre de nombreuses controverses doctrinales. Les codes de conduite sont dépourvus de force obligatoire et sont appelés à jouer un rôle de «persuasion morale ». Nous retiendrons simplement que les codes de conduite et les labels sont véritablement des outils d'autorégulation au sens large du terme, puisque les auteurs de la norme en sont également des destinataires. Il est alors nécessaire de se concentrer sur la dimension pratique ou encore l'effectivité de cette autorégulation, c'est-à-dire sur la capacité des codes de conduite à influencer le comportement des sociétés transnationales, d'autant plus que, comme le remarque FARJAT (2005, p.76), « l'effectivité d'une norme n'est pas nécessairement liée à son obligation ». Et « le respect de la loi n'est que rarement dû à la crainte de la sanction mais découle plutôt du concept de "respect volontaire », c'est-à-dire le respect de la réglementation en raison de convictions personnelles ou de pressions sociales » (2005, p.76).

Selon CHEVALIER (2003, p. 90 s.), « le phénomène de l'Union européenne est particulièrement sensible à une telle évaluation post-moderne du droit, dans la mesure où il est intrinsèquement lié à la crise de l'Etat Nation, seule source de légitimité du droit moderne ». La reconnaissance d'une place substantielle aux ONG et plus généralement à la société civile dans la gestion des affaires publiques européennes s'inscrit dans une logique de déstructuration de la confrontation hégélienne entre l'Etat et la société.

Rappelons la théorie de l'intégration «Integrationslehre », proposée par SMEND (1928), qui appréhende la fonction du droit comme un processus de régulation et d'expression des faits sociaux et politiques. Le droit ne se présente pas comme une alternative à une Gouvernance " politique » et vice versa. La norme de droit se crée et s'applique comme condition et produit du processus politique. D'autre part, et tout particulièrement pour les systèmes de normes juridiques à vocation « constituante », la théorie de l'intégration essaie de rationaliser le politique et le social - qui génèrent des tendances intrinsèquement centrifuges et potentiellement radicales - autour des standards, appelés «valeurs » qui forment la mesure des attitudes politiques. En somme, le droit offre la plateforme pour la prise de décision, une décision qui met en marche le système politique et suggère, par rétroaction, la création de droit nouveau.

Qui fait quoi ? A ce propos, les mots prononcés par Jean MONNET lors d'un séjour à Londres en 1973 trouvent encore aujourd'hui toute leur acuité : « Il est indispensable que l'objectif politique poursuivi par la Communauté soit clairement expliqué et que la méthode de travail commune soit connue. L'opinion publique ne sera touchée au cœur que si elle sait qu'une Autorité européenne existe et qu'il n'y a pas seulement des technocrates, ce qui est le cas aujourd'hui. Des institutions européennes sortiraient une autorité. Nous devons reconnaître que ce n'est pas le cas. Quelles qu'en soient les raisons, la forme administrative des résolutions, les lenteurs est les complexités techniques lassent le public. Les institutions ont été indispensables et le sont encore, mais elles ne correspondent pas aujourd'hui à la nécessité d'aller vite et de décider» (FONTAINE, 1988, p. 116).

Les acteurs non étatiques y endossent plusieurs rôles, tantôt relais locaux d'information et de surveillance, tantôt agents de mise en œuvre directe ou indirecte des normes à l'émergence desquelles ils participent.

La diversité et la multiplication des acteurs, institutionnels et privés, participant à ce contrôle tendent à conférer à la décision finale une indéniable légitimité. Mais cette acceptabilité 
reste largement conditionnée par la participation effective de chacun au processus décisionnel et juridictionnel. La place des différents acteurs varie au cours des principales phases du contrôle et les interventions tendent à diminuer la portée du contrôle exercé par l'acteur décisionnel principal, la Commission, au profit de l'émergence de nouveaux acteurs, le juge et les tiers.

\section{CONCLUSION}

Cette analyse des concepts de gouvernance et de mondialisation, dans cet article, dépasse effectivement l'idée polysémique que l'on s'en fait habituellement.

Bien que le terme de mondialisation soit souvent utilisé de façon péjorative (résistances et inégalités dans le monde, contestations altermondialistes etc..), il répond pourtant, sous son triple aspect économique, social et politique à une éthique de liberté universelle : l'échelle mondiale de réussite. En effet, un ordre économique mondial, fondé sur la liberté des échanges, doit accompagner un ordre politique mondial, manifesté par l'horizon démocratique et fondé sur la liberté des citoyens. Par ailleurs, la mondialisation ne se confond pas avec le processus de modernisation car elle en n'est qu'un moment particulier et ne rime pas obligatoirement avec uniformisation, à l'instar de la société japonaise. La modernité est donc un véritable moyen de compréhension de la mondialisation.

Face à cette mutation de la société internationale, la mondialisation ne met pas fin aux singularités culturelles et sociales, elle leur donne au contraire de nouvelles scènes, de nouveaux masques et de nouveaux statuts.

De plus, les possibilités d'une gouvernance mondiale ont également été analysées. Par le remodelage de la scène internationale, la multiplicité d'acteurs, le processus normatif international apparaît, aujourd'hui, comme le fruit d'incessantes et d'inévitables négociations, notamment les acteurs non étatiques au fonctionnement de l'organisation internationale. Aujourd'hui, la régulation est porteuse de flexibilité et de souplesse dans l'encadrement juridique des activités et des situations : on assiste à un accroissement exponentiel d'instruments de soft law (« droit mou »). La viabilité du phénomène « de régulation » est liée à un défi majeur : celui de sa légitimité. Les principes de non exclusion, de participation publique, d'accès à l'information ou d'équilibre pourraient garantir une régulation plus transparente et plus démocratique. Par exemple, les initiatives les plus intéressantes des régulations environnementale ou sociale d'origine privée sont caractérisées par l'élaboration de codes de conduite ou autres labels. C'est le phénomène de l'autorégulation. En conclusion, sur le plan de la gouvernance mondiale, la diversité et la multiplication des acteurs (institutionnels et privés) renforcent la légitimité de la régulation, mais cette acceptabilité reste largement conditionnée par la participation effective de chacun de ces acteurs au processus décisionnel et juridictionnel.

\section{BIBLIOGRAPHIE}

ABI KHALIL, R., Mondialisation et gouvernance mondiale : quelles perspectives, L'Harmattan, (Livre numérique) 2015. BEVIR, M., Democratic Governance. Princeton, N.J. : Princeton University Press, 2010.

BEVIR, M., Democratic Governance: A Genealogy. Local Government Studies, 37:1, 3-17, 2011.

BEVIR, M., Democratic Governance: Systems and Radical Perspectives. Public Administration Review, 66:3, 426-436, 2006.

BEVIR, M., Democratic governance: History, practice, reality and possibility. British Politics, 6:2, 273-283, 2011. 
BOQUET, Yves. La mondialisation. Un monde organisé en systèmes. Dijon, Editions Universitaires de Dijon, 135 p., 2018.

CARROUÉ, Laurent. Atlas de la mondialisation. Une seule terre, des mondes. Paris, Autrement, 95 p. 2018.

CHAZOURNES L. Boisson de, ROSTANE M. Une société internationale en mutation : quels acteurs pour une nouvelle gouvernance ? dans les Travaux du CERIC - Collection Dirigée par Jacques Bourrinet. Bruylant, Bruxelles, 2005.

CHENET, François. La mondialisation vue de l'Inde. in La mondialisation entre illusion et utopie. Archives de philosophie du droit - Tome 47 - Ouvrage publié avec le concours du CNRS. Dalloz, 2003.

CHEVALLIER, Jacques. L'Etat post-moderne. LGDJ (Droit et Société), Paris, 2003.

DE SALLE, Corentin. Fin de l'histoire et Légitimité du Droit dans l'œuvre de F.A.von Hayek, in La mondialisation entre illusion et utopie. Archives de philosophie du droit - Tome 47 - Ouvrage publié avec le concours du CNRS. Dalloz, 2003.

DEFARGES, Philippe Moreau. La réforme des institutions internationales. Archives de philosophie du droit. Tome 47 - Ouvrage publié avec le concours du CNRS. Dalloz, 2003.

FARJAT, Gérard. Réflexion sur les codes de conduite privés, in Le droit des relations économiques internationales, Etudes offertes à Berthold Goldman, Litec, Paris, 1982. FONTAINE, Pascal. Jean Monnet - L’inspirateur. Persée, Paris, 1988.

FRISON-ROCHE, Marie- Anne. Le droit des deux mondialisations. in La mondialisation entre illusion et utopie. Archives de philosophie du droit - Tome 47 - Ouvrage publié avec le concours du CNRS. Dalloz, 2003.

GEORGE, S. Vivre avec gaieté dans un monde tragique, in La sagesse d'aujourd'hui. Paris, 2002.

KAMTO, M. Gouvernance mondiale et droit international. Bruxelles: Bruylant (Organisation internationale et relations internationales, 79), 2015.

LAÏDI, Zaki. Les enjeux de la gouvernance mondiale, en ligne :

www.diplomatie.gouv.fr/fr/IMG/pdf/FD001432.pdf.

LE CACHEUX, Jacques. Mondialisation économique et financière : de quelques poncifs, idées fausses et vérités, in Revue de l'OFCE 2002/5 (nº 83 bis), 2002.

LEPELTIER, Serge. Mondialisation : une chance pour l'environnement ? Rapport d'information. $n^{\circ} 233,2004$.

MATTEI, Jean-François. Monde, mondialisation et liberté, in La mondialisation entre illusion et utopie. Archives de philosophie du droit - Tome 47 - Ouvrage publié avec le concours du CNRS. Dalloz, 2003. 
OHMAE, Kenichi. The Bordless World : power and strategy in the interlinked economy., London: Fontana, 1990.

PERELMAN, Chaïm. Logique juridique. La nouvelle rhétorique, Dalloz, 1979, rééd. 1999. SÈVE, RENÉ. La mondialisation entre illusion et utopie. Archives de philosophie du droit Tome 47 - Ouvrage publié avec le concours du CNRS. Dalloz, 2003.

SLOTERDIJK, Peter. La mobilisation infinie. Paris : Bourgeois, 2000.

SMEND, Rudolf. Verfassund und Verassungsrecht, Duncker \& Humblot, Berlin, 1928.

WARNIER Jean-Pierre, La Mondialisation de la culture. Paris, 1999.

WEIL, Prosper. Le droit international économique : Mythe ou réalité, in Aspects du droit international économique, SFDI, Colloque d'Orléans, édition Pedone, Paris, 1972.

WILLIAMSON O., Markets and hierarchies. Analysis and antitrust implications., New York: Free Press, 1975.

YATABE, Kazuhiko. La mondialisation vue du Japon.. in La mondialisation entre illusion et utopie. Archives de philosophie du droit - Tome 47 - Ouvrage publié avec le concours du CNRS. Dalloz, 2003. 